\title{
Research priorities for COVID-19 sensor technology
}

To the Editor - The COVID-19 pandemic has spurred efforts to develop sensor technology to manage the disease ${ }^{1-3}$. Most of these projects have been driven by medical researchers, scientists and engineers without explicit involvement and input from patients and the broader community. Here, we define sensor technology broadly to include physical, cellular and molecular platforms that produce signals to identify specific events associated with SARS-CoV-2 and/or its interaction with the host. The main applications of sensor technology in COVID-19 have been to detect fever using infrared sensing devices and the presence of viral RNA using polymerase chain reaction (PCR) tests ${ }^{1}$. However, a substantial proportion of individuals with COVID-19 never develop fever ${ }^{1}$. PCR tests have been developed to detect SARS-CoV-2 in nasopharyngeal samples, but to date they have been expensive, resource-intensive, cumbersome and relatively slow. Moreover, positive PCR tests do not imply a person is still infectious and thus have not provided information about transmissibility or virulence $^{1,4,5}$, hampering the development of more effective action plans in the societal, economic and public health dimensions ${ }^{6}$.

Given the urgent need to better control the pandemic and its impact on the community, resources should be allocated in a strategic and targeted manner that takes into account community perspectives, through an explicit consensus-based process with equitable involvement of patients, the public, researchers and clinicians. Co-production in research specifically involving consumers or end-users is now widely advocated to improve the relevance, use and impact of the findings ${ }^{7,8}$. It requires partnership and collaboration between researchers and the broader community from the outset, beginning with priority-setting. There have been few research priority-setting partnerships in COVID-19, with very few involving patients and the public, and none with a focus on sensor technology. Below, we describe the development and outcome of a process through which we identified the shared priorities of patients, the community, health professionals, scientists, engineers and policy makers for research in sensor technology to address COVID-19, the reasons for their priorities, and ideas for implementation.
This priority-setting project involved 83 patients with COVID-19, family members, the general public, scientists, engineers, health professionals (including specialist clinicians from multiple disciplines, such as infectious diseases, diagnostic pathology, cardiology or cardiovascular diseases, respiratory medicine, geriatrics, emergency medicine, critical care medicine, gastroenterology, hematology, pediatrics, infection prevention and control, and digital health), policy makers, industry representatives and funders.

We conducted an online survey to prioritize research statements in which respondents $(n=43)$ rated their importance using a 9-point Likert scale (7-9 indicating 'critical importance'). The mean score, median and proportion of participants who rated the statement to be critically important are provided in Supplementary Table 1. Research statements that had a mean and median of $\geq 7$ were discussed at a consensus workshop, conducted using Zoom videoconferencing on 20 August 2020, with the following goals: to achieve agreement on the research priorities, generate ideas for sensor technologies and discuss facilitators and barriers to implementation. To encourage diverse discussions, the 65 attendees were preassigned to six virtual breakout groups, with each group including patients who had been diagnosed previously with COVID-19 and/or family members, health professionals, scientists or engineers, and policymakers or funders. Each breakout group was managed by a facilitator and cofacilitator who moderated the discussion using the workshop question guide (Supplementary Table 2). All discussions were transcribed. We identified reasons for the priorities, ideas for sensor technologies (compounds, devices, general application), and the implementation of each (feasibility, usability, acceptability).

Of the 18 research statements, 8 had a mean and median score of 7 or more (Table 1). The top three priorities were the following: develop a point-of-care screening test for COVID-19; detect how contagious a person with COVID-19 is; and identify the level of immunity a person has to COVID-19. The reasons for priorities were summarized in four themes. First, 'Enabling more efficient clinical decision-making' was driven by the need to prevent delays in access to treatment, preserve finite resources (in terms of staffing, facilities for quarantine and personal protective equipment) and to provide prognostic information to inform patient care. Second, 'Minimizing societal disruption' was emphasized to enable a return to normal life and to reduce stigma and isolation. Third, 'Protecting the community' supported the need for sensor technology that could trigger contact tracing, establish safe environments, safeguard the vulnerable, gauge individual susceptibility to COVID-19, and manage the risk among healthcare workers. And finally, 'Preparedness for the next phase of the pandemic' required sensor technology to be relevant and responsive to the development of immunity and vaccines, and to help maintain the suppression phase over the long term. A detailed description with supporting quotations for each theme is provided in Supplementary Table 3.

For each of the top research priority statements, the specific suggestions for sensor technology (including compounds and devices) and its application are summarized in Table 1 . The suggestions of ensuring feasibility, usability and acceptability of sensor technology and applications to address COVID-19 are outlined in Supplementary Table 4 . These have been identified as essential attributes for an ideal sensor for pandemics in general, including accuracy, a fast response time, multiplexing capabilities, multiple sensing modes (sensor fusion and the use of artificial intelligence to detect signatures that reveal infection), disposability, long shelf life, ease of use, cost-effectiveness, manufacturability, and autonomy ${ }^{2}$. Particular emphasis was placed on the need for samples to be easy and safe to collect and the need for sensor devices to be non-invasive and their use regulated appropriately to ensure data privacy. The legal, ethical and privacy concerns surrounding the use of digital technology in COVID-19 are highly relevant given the need for public trust and engagement to ensure widespread uptake ${ }^{1}$.

Patients in particular emphasized the profound impacts of COVID-19 on mental health as a consequence of self-isolation and quarantine. Specifically, patients gave high priority to the detection of immunity and wanted assurance that they were no longer contagious because families and friends were 
Table 1 | Suggestions and ideas for sensor technology to address COVID-19

Statement
Develop a point-of-care (instant)
screening test for COVID-19

Detect how infectious a person with a virus is

Identify the level of immunity a person has to COVID-19, or its change over time

Develop tests to assess people's immunity against the virus

Identify who needs a COVID-19 test in individuals who are asymptomatic (do not have symptoms)

Develop a non-invasive, quick, cheap, and effective diagnostic test that people can do themselves

Develop a detector for airborne virus

Identify who needs a COVID-19 test in people who have a chronic condition and with symptoms similar to COVID-19 (for example, persistent cough)

\section{Ideas for compounds, devices and general application}

- Target a different type of sample or organ (other than blood, nasal or throat swab, or temperature), for example, urine (non-aerosol-generating procedure)

- Detect other compounds or chemicals that the body generates due to infection and that may be used as a signature of disease

- Detect viral components

- Use microfluidic technology: capture particles on a microfluidic system on a chip; they go to the optic sensor and the optic sensor functions as a screener

- Develop wearable non-intrusive devices for healthcare workers that capture temperature and other clinical parameters, with data captured in a central service for monitoring, and use artificial intelligence to analyze the data

- Create a device used in the toilet to measure microbiome, with data connected to a phone or other device; sample viral loads in sewage

- Support rapid transmission of data packets

- For individual screening, offer conventional methods such as a strip and a reader, with results transmitted to a central location

- Develop a dipstick test using saliva

- Analyze physiological or biomarker responses (similarly to browsers that assess whether people are robots) with artificial intelligence machine learning

- Use a microphone to detect characteristics of breathing

- Provide a device to monitor hypoxia

- Develop a device to monitor the ability to smell, olfactory type of sensing

- Provide a device to monitor heart rate

- Use sensors to detect inflammation in the mucosal tract

- Develop a mask with breath analysis to signal infection

- Develop face masks with sensors (for example, one that changes color if a person is contagious)

- Use sensors to measure breathing, cough, inflammation

- Develop a tongue swab to determine viral load on site

- Develop cell cultures with cell types that are very susceptible infection by the virus, and expose them to infected people to measure transmissibility

- Measure the aerosol and droplet release that come from talking, sneezing, coughing, perspiration; quantify the particle release from a person and their interaction with other people nearby

- Use digital imaging processing to detect particle exchange

- Develop a saliva test that uses spike protein as a capture medium for immunoglobulin

- Develop a microneedle patch-based device that may be inserted under the skin in the arm (similar to ones used for glucose monitoring in patients with diabetes) for ongoing monitoring

- Identify signals of immunity from, for example, sweat, breath

- Measure serological responses, T cell responses

- Use surveillance technology such as infrared sensors or camera networks and wearables to pick up infected people (who may not exhibit traditional symptoms) by temperature, heat mapping, changes in heart rate, blood pressure, sounds or other observables

- Monitor sewage systems

- Develop breath analysis devices that can detect viral particles

- Use blood samples (similarly to a glucose monitor)

- Use respiratory secretions for a home test

- Develop a urine-based test (similar to a pregnancy test)

- Use detectors in ventilation systems to detect viruses in crowded or high-risk places (for example, nursing homes, supermarkets)

- Connect detectors to an alert system (one that does not cause panic)

- Use animals such as trained dogs to detect COVID-19

- Detect the virus using a physical process, for example, an integrated device that works with light as the virus absorbs and scatters light

- Develop a device for the room or environment (rather than for people)

- Develop a device to sense variability in heart rate, skin temperature; that is, change in biomarkers avoiding them for an indefinite period of time (for some, over six months), given the fear and stigma attached to COVID-19.
Developing sensor technology for the detection of protective immunity is also important, given the imminent distribution of vaccines and uncertainty regarding long-term immunity and reinfection ${ }^{3,9,10}$. Detection of immunity can further 


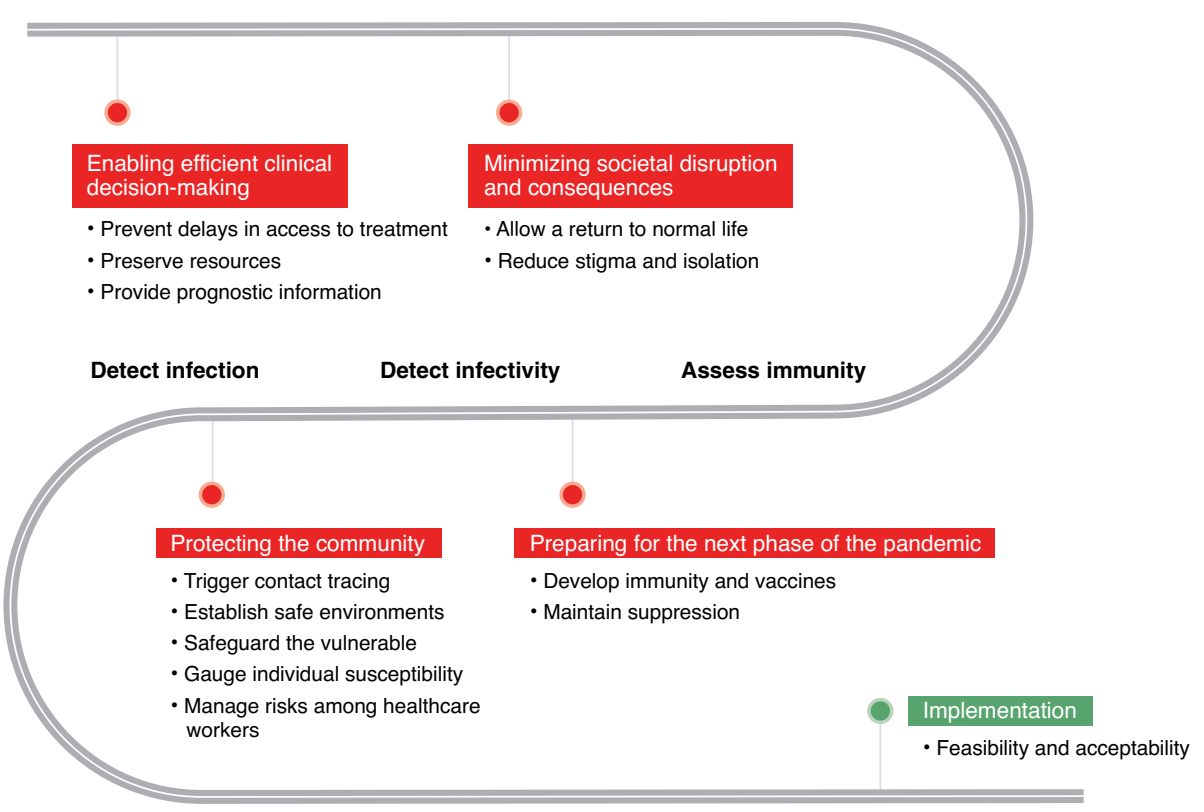

Fig. 1| A roadmap for research priorities for COVID-19 sensor technology.

inform strategies to minimize societal and economic disruption. Of note, physiological monitoring was perceived to be lower in priority compared with rapid diagnosis and assessment of immunity, despite the possibility of its detecting early changes in clinical status that require rapid intervention or admission to hospital (that is, its potential as a screening tool).

The research priorities were motivated by personal and altruistic concerns. These included anxiety and concern about the need for urgency in diagnosis vis-à-vis impact of diagnostic delays on the severity and spread of COVID-19, a uniform preference for convenient sample types for testing, and the personal and public health need to be confident in the validity of markers of protective immunity and the duration of such immunity in response to either natural infection or vaccination. The high priority that patients gave to measuring infectivity and immunity to address stigma, fears and rejection they encountered from community members when told of their COVID-19 diagnosis would not have been considered without inclusion of this stakeholder group in the prioritization process.

Further work is required to identify the extent to which these priorities can be generalized globally or in specific settings, such as in low-resource settings or countries, where access to healthcare is limited. Furthermore, immediate research priorities may differ in countries, locations or cultures experiencing different stages of the pandemic. Nevertheless, collectively, such studies can help to formulate an equitable and coordinated international response to future pandemics and other global threats, including antimicrobial resistance, and mitigate the threat of such crises on people's lives and the world's economy (Fig. 1).

Advances in nanotechnology and the Internet of things have stimulated the proliferation and ubiquity of sensor technology ${ }^{2}$. Traditionally, advances in sensor research have been the province of experts in smart technology and have not systematically and explicitly included the perspectives of the ultimate end users or beneficiaries. Although consideration is given to the end user in the design of sensor technology, the process is usually linear and between only two stakeholder groups. The inclusion of diverse stakeholder groups provides a more complete perspective to support uptake. Coproduction brings in the human context and attention to the areas of greatest importance, which are underpinned by social, ethical and human dimensions beyond just technical considerations. We believe this process provides a roadmap for the allocation of resources to purposefully advance sensor technology, which will strengthen the whole of society's response to the COVID-19 pandemic and enhance preparedness for future pandemics.

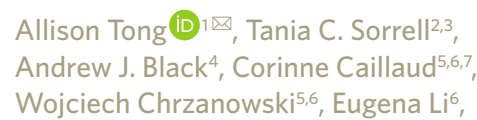

David Martinez-Martin (D) 6,8 ,

Alistair McEwann, ${ }^{6,8}$, Rex Wang ${ }^{6}$,

Alice Motion (D) 6,9,

Alvaro Casas Bedoya ${ }^{6,10}$, Jun Huang 6,8 Lamiae Azizi ${ }^{6,11}$, Benjamin J. Eggleton (D) 6,10 and The COVID-19 Sensor Research

Priority-Setting Investigators*

${ }^{1}$ Sydney School of Public Health, The University of Sydney, Sydney, NSW, Australia. ${ }^{2}$ Marie Bashir Institute for Infectious Disease and Biosecurity, The University of Sydney and Westmead Institute for Medical Research, Sydney, NSW, Australia. ${ }^{3}$ Sydney Medical School, The University of Sydney, Sydney, NSW, Australia. ${ }^{4}$ Westmead Living Lab, The University of Sydney, Sydney, NSW, Australia. ${ }^{5}$ Charles Perkins Centre, The University of Sydney, Sydney, NSW, Australia. ${ }^{6}$ The University of Sydney Nano Institute (Sydney Nano), The University of Sydney, Sydney, NSW, Australia. ${ }^{7}$ School of Medical Sciences, The University of Sydney, Sydney, NSW, Australia. ${ }^{8}$ School of Biomedical Engineering, The University of Sydney, Sydney, NSW, Australia. ${ }^{9}$ School of Chemistry, The University of Sydney, Sydney, NSW, Australia. ${ }^{10}$ School of Physics, University of Sydney, Sydney, NSW, Australia. ${ }^{11}$ School of Mathematics and Statistics, The University of Sydney, Sydney, NSW, Australia. ${ }^{*} A$ list of authors and their affiliations appears at the end of the paper.

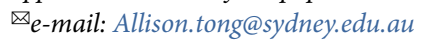

Published online: 18 January 2021 https://doi.org/10.1038/s41587-021-00816-8

References

1. Budd, J. et al. Nat. Med. 26, 1183-1192 (2020).

2. Bhalla, N., Pan, Y., Yang, Z. \& Payam, A. F. ACS Nano 14, 7783-7807 (2020).

3. Koff, W. C. \& Williams, M. A. N. Engl. J. Med. 383, 804-805 (2020).

4. Udugama, B. et al. ACS Nano 14, 3822-3835 (2020).

5. Nagura-Ikeda, M. et al. J. Clin. Microbiol. 58, e01438-20 (2020).

6. Tom, M. R. \& Mina, M. J. Clin. Infect. Dis. 71, 2252-2254 (2020).

7. Anonymous. Nature 562, 7 (2018)

8. Hickey, G., Richards, T. \& Sheehy, J. Nature 562, 29-31 (2018).

9. Chaturvedi, R., Naidu, R., Sheth, S. \& Chakravarthy, K. Disaster Med. Public Health Prep. https://doi.org/10.1017/dmp.2020.216 (2020).

10. Gousseff, M. et al. J. Infect. 81, 816-846 (2020).

\section{Acknowledgements}

The project is funded by Sydney Nano. A.T. is supported by the University of Sydney Robinson Fellowship and a National Health and Medical Research Council Investigator Grant (1197324). The funding organizations had no role in the design and conduct of the study; collection, management, analysis and interpretation of the data; or preparation, review or approval of the manuscript. We acknowledge, with permission, all the attendees who participated in the research priority-setting workshop: Sarah Al-Horani, Tomas Andersen, Rodney Aggett, Lamiae Azizi, Andrew Baillie, Andrew Black, Celine Boehm, Philip Britton, Anthony Brown, Mitchell Burger, Corinne Caillaud, Alvaro Casas Bedoya, Steve Chadban, Elaine Chan, Nanda Sakaleshpura Chandrashekar, Gael Clerc, Wojciech Chrzanowski, Ben Eggleton, Simon Fleming, Gregory Fox, Luke Gordon, Nicholas Haskins, Anita Ho-Baillie, Martin Howell, Jun Huang, Nicholas Hunt, Owen Hutchings, Jonathan Iredell, Garry Jennings, Craig Jin, Omid Kavehei, Paris Kilham, Leonard Kritharides, Ben Kwan, Sergio Leon-Saval, Eugena Li, Steven Maguire, David Martinez-Martin, Annie McCluskey, Alistair 
McEwan, Nicholas McKay, Julian Morgans, Alice Motion, Stefano Palomba, Alan Pettigrew, Svetlana Postnova, Cathy Quinlan, James Rabeau, Katie Richmond, Damien Rothstein, Nicole Scholes-Robertson, Tim Shaw, Vitali Sintchenko, Thomas Snelling, Tania Sorrell, Allison Tong, Alessandro Tuniz, Pegah Varamini, Audrey Wang, Kailing Wang, Rex Wang, Alexander Witherden, Benjamin Wright, Wilson Yeung and Hans Zoellner. We acknowledge and pay respect to the Gadigal people of the Eora Nation, the traditional owners of the land on which we research, teach and collaborate at The University of Sydney.

Competing interests

The authors declare no competing interests.

\section{Additional information}

Supplementary information The online version contains supplementary material available at https://doi.org/

10.1038/s41587-021-00816-8.

The COVID-19 Sensor Research

Priority-Setting Investigators

Sarah Al-Horani'2, Tomas Andersen ${ }^{13}$,

Rodney Aggett ${ }^{14}$, Lamiae Azizi ${ }^{13}$,

Andrew Baillie ${ }^{15}$, Alexandra Barratt ${ }^{13}$,

Andrew Black ${ }^{13}$, Celine Boehm ${ }^{13}$, Philip Britton ${ }^{16}$,

Anthony Brown ${ }^{17}$, Mitchell Burger ${ }^{18}$,

Corinne Caillaud ${ }^{13}$, Alvaro Casas Bedoya ${ }^{13}$,

Steve Chadban ${ }^{19}$, Elaine Chan ${ }^{20}$,

Nanda Sakaleshpura Chandrashekar ${ }^{21}$,

Clara Chow $^{13}$, Gael Clerc ${ }^{22}$,

Wojciech Chrzanowski ${ }^{13}$, Chris Douglas ${ }^{14}$,
Benjamin Eggleton ${ }^{13}$, Simon Fleming ${ }^{13}$, Gregory Fox ${ }^{13}$, Nicky Gilroy ${ }^{20}$, Luke Gordon ${ }^{13}$, Nicholas Haskins ${ }^{23}$, Anita Ho-Baillie ${ }^{13}$, Martin Howell ${ }^{13}$, Ian Hickie ${ }^{13}$, Jun Huang ${ }^{13}$, Nicholas Hunt ${ }^{13}$, Owen Hutchings ${ }^{24}$, Jonathan Iredell ${ }^{25}$, Garry Jennings ${ }^{26}$, Craig Jin $^{13}$, Kristina Kairaitis ${ }^{13}$, Omid Kavehei ${ }^{13}$, Peter Kennedy ${ }^{27}$, Paris Kilham ${ }^{14}$, Leonard Kritharides ${ }^{13}$, Ben Kwan ${ }^{28}$, Sergio Leon-Saval' ${ }^{13}$, Eugena $\mathrm{Li}^{13}$, Richard Lindley ${ }^{13}$, Deena Lynch ${ }^{29}$, Steven Maguire ${ }^{13}$, David Martinez-Martin ${ }^{13}$, Annie McCluskey ${ }^{13}$, Alistair McEwan ${ }^{13}$, Nicholas McKay ${ }^{13}$, Geoffrey Mifsud ${ }^{30}$, Julian Morgans ${ }^{31}$, Alice Motion ${ }^{13}$, Stefano Palomba ${ }^{13}$, Alan Pettigrew ${ }^{13}$, Svetlana Postnova ${ }^{13}$, Joseph Prinable ${ }^{32}$, Cathy Quinlan ${ }^{33}$, James Rabeau ${ }^{13}$, Mark Rees ${ }^{13}$, Katie Richmond ${ }^{13}$, Damien Rothstein ${ }^{14}$, Richard Savoie ${ }^{34}$, Nicole Scholes-Robertson ${ }^{13}$, Ian Seppelt ${ }^{13}$, Tim Shaw ${ }^{13}$, Vitali Sintchenko ${ }^{13}$, Thomas Snelling ${ }^{16}$, Tania Sorrell ${ }^{13}$, Armando Teixeira-Pinto ${ }^{13}$, Allison Tong ${ }^{16}$, Euan Tovey ${ }^{13}$, Alessandro Tuniz ${ }^{13}$, Pegah Varamini ${ }^{13}$, Kavita Varshney ${ }^{20}$, Audrey P. Wang ${ }^{13}$, Kailing Wang ${ }^{13}$, Rex Wang ${ }^{13}$, Steven Wise ${ }^{13}$, Alexander Witherden ${ }^{35}$, Benjamin Wright ${ }^{36}$, Wilson Yeung ${ }^{21}$ and Hans Zoellner ${ }^{13}$
${ }^{12}$ University of Technology, Ultimo, NSW, Australia.

${ }^{13}$ The University of Sydney, Camperdown, NSW, Australia. ${ }^{14}$ Sydney, NSW, Australia. ${ }^{15}$ The University of Sydney, Sydney Local Health District, Camperdown, NSW, Australia. ${ }^{16}$ The University of Sydney, The Children's Hospital at Westmead, Westmead, NSW, Australia. ${ }^{17}$ Health Consumers NSW, Sydney, NSW, Australia. ${ }^{18}$ Sydney Local Health District, Sydney, NSW, Australia. ${ }^{19}$ The University of Sydney, Royal Prince Alfred Hospital, Camperdown, NSW, Australia. ${ }^{20}$ Westmead Hospital, Westmead, NSW, Australia. ${ }^{21}$ eHealth NSW, Sydney, NSW, Australia. ${ }^{22}$ Bepatient, Sydney, NSW, Australia. ${ }^{23}$ NSW Smart Sensing Network, Sydney, NSW, Australia. ${ }^{24}$ Royal Prince Alfred Hospital, Camperdown, NSW, Australia. ${ }^{25}$ The University of Sydney, NSW Health Pathology, Westmead Institute for Medical Research, Westmead, NSW, Australia. ${ }^{26}$ Sydney Health Partners, Camperdown, NSW, Australia. ${ }^{27}$ eHealth NSW, Chatswood, NSW, Australia. ${ }^{28}$ Sutherland Hospital, Caringbah, NSW, Australia. ${ }^{29}$ Jonze Society, Brisbane, QLD, Australia. ${ }^{30}$ The University of Sydney, Westmead Hospital, Westmead, NSW, Australia. ${ }^{31}$ Vice, Melbourne, VIC, Australia. ${ }^{32}$ The University of Sydney, ACRF Image X Institute, Camperdown, NSW, Australia. ${ }^{33}$ Royal Children's Hospital, Parkville, Victoria, Australia. ${ }^{34}$ Adionatech, Sydney, NSW, Australia. ${ }^{35}$ Camperdown, NSW, Australia. ${ }^{36}$ Nanosonics Ltd, Sydney, NSW, Australia.

\section{Will Ethiopia be a springboard or a stonewall for GM crops in Africa?}

To the Editor - As a systems agronomist with substantial experience in the Consortium of International Agricultural Research centers (CGIAR) and national research institutions in sub-Saharan Africa, I have followed with interest the recent controversy around plantings of transgenic crops in Ethiopia. Until 2015 , the country took a vocal stand against genetically modified (GM) crops, underlined by its strict proclamation on biosafety in 2009 (Proclamation No. $655 / 2009)^{1}$. The regulation was so inflexible that a special permission was required to transit any "modified organisms" through Ethiopian customs. Six years later, the country loosened its restrictions in an amended proclamation (Proclamation No. $896 / 2015)^{2}$. The latter proclamation allows the commercial cultivation of genetically modified (GM) cotton and confined field research on GM maize and enset (Ensete ventricosum), a food plant whose cultivation is endemic to Ethiopia. As a result, Bt-cotton has been under widespread production and the country has lately issued a five-year permit to conduct confined field trails on drought-tolerant and pest-resistant GM maize $^{3}$. GM maize trails were successfully conducted in 2019 by the Ethiopian Institute of Agricultural Research ${ }^{4}$.

In a recent report ${ }^{4}$, the US Department of Agriculture (USDA) recognized Ethiopia's commitment to implementing the amended protocol. Although debates about gene-modified organisms (GMOs) in Ethiopia started immediately after the first prohibitive proclamation in 2009, they were low-key and mostly pro-GMO (Fig. 1). Severe criticisms against GMOs exploded following USDA's accolades for Ethiopia's relaxation of rules ${ }^{5-7}$
As debates intensified following the USDA report, rather than Ethiopia's decision per se, one explanation is that the controversy is driven by paranoia that the United States is using Ethiopia as biotech strategic entry point to expand its GMO portfolio in Africa. Anti-biotech activists often amplify these kinds of strong rhetorical statements ${ }^{8}$, which have a potential to entrench throughout the continent. As regulatory systems in most African countries are grappling with the GMO dilemma, Ethiopia's final regulatory stance on biotech products will have broader implications. Given Ethiopia's diplomatic muscle in the region, this forms a turning point for the fate of biotech products all over the continent and beyond. Because Ethiopia is Africa's diplomatic epicenter, its endorsement or dismissal of GMOs may 